\title{
Phenylketonuria in a low incidence population: molecular characterisation of mutations in Finland
}

Per Guldberg, Karen Friis Henriksen, Ilkka Sipilä, Flemming Güttler, Albert de la Chapelle

\begin{abstract}
The incidence of phenylketonuria (PKU) in Finland is extremely low, probably below 1 in 100000 . We describe the mutations and haplotypes in all four presently known patients. Mutation $\mathrm{R408W}$ was found on four mutant chromosomes (all haplotype 2), and IVS7nt1, R261Q, and IVS2nt1 were each found on a single chromosome. No mutation was found on the remaining chromosome. These findings support a pronounced negative founder effect as the cause of the low incidence of PKU in Finland, and are consistent with existing data regarding the European and Baltic origin of Finnish genes.
\end{abstract}

( $(\mathcal{M}$ Med Genet 1995;32:976-978)

Studies are being conducted to determine the origin and spread of the different mutations of the phenylalanine hydroxylase (PAH) gene that cause phenylketonuria (PKU). Of particular interest is the prevalent $\mathrm{R} 408 \mathrm{~W}$ mutation. Based on haplotype data, this mutation is now believed to have arisen at least twice, once in western Europe (on haplotype 1) and once in eastern Europe (on haplotype 2) $1{ }^{2}$ Finland is located at the northern border between east and west in Europe so the distribution of mutations and haplotypes might be expected to have both eastern and western characteristics. Yet no molecular data on PAH mutations have so far been published. This is because of the extremely low incidence of PKU in Finland. The first patient diagnosed in Finland was found by screening 509 institutionalised people with severe mental retardation. ${ }^{3}$ Further attempts to screen for PKU in Finland were reported by $\mathrm{Palo}^{4}$ who found two patients among a total of 2090 mentally retarded subjects. Visakorpi et al screened a further 1595 mentally retarded people without finding any PKU patients. These data suggested a prevalence of 0.54 per 1000 among the severely mentally retarded, a figure less than one-tenth of the corresponding figures in northern European countries studied by similar methods. ${ }^{6}$

To settle the question of the incidence of PKU in Finland, Visakorpi $e t a \bar{l}$ undertook state of the art screening of random newborns by the Guthrie test. After 71135 babies had been screened without a single case of PKU being found, the screening was stopped in
1970 , and it was concluded that the incidence of PKU in Finland is less than 1 per $100000 .^{5}$

Observations after 1970 have borne out this assumption. As PKU is not screened for in Finland, and as there are no exclusive clinical symptoms or signs suggesting the disease in an infant, new cases will inevitably go undetected initially. However, in the highly centralised Finnish medical care system, they are unlikely to escape detection later because of developmental delay and severe mental retardation. This is because all mentally retarded people are subjected to a variety of diagnostic procedures, including screening for metabolic disorders, on more than one occasion before adulthood. Over the past decade we have made efforts to ascertain all cases of PKU in Finland. As a result, a total of four living PKU patients are known to us. As Finland's population is 5 million and the annual number of births is approximately 70000 , this small number is consistent with an incidence of PKU well below the 1 in 100000 mentioned above. Thus Finland's frequency of PKU is remarkably low, being less than one-tenth of the overall frequency among white populations. ${ }^{7}$

We report here haplotype and mutation analyses in the four known Finnish PKU patients. The study was undertaken with the aim of determining the types and origins of the mutations and the mechanisms that have led to the low incidence.

\section{Materials and methods PATIENTS}

Four living Finnish subjects have been diagnosed with PKU and are known to us. Patient LGL 5044, born in 1958, was found to have raised urinary phenylalanine at 8 years. Her plasma phenylalanine levels are not known. She is severely retarded and most probably has classical PKU. Patient LGL5072, born in 1979 , was diagnosed at 13 months with plasma phenylalanine levels of more than $3 \mathrm{mmol} / \mathrm{l}$ and has classical PKU. The patient responded well to a phenylalanine restricted diet and at 16 years of age attends normal school (with considerable difficulties). Patient LGL5741, born in 1986, was noted to have severe developmental delay as an infant, but amino acid analyses were not done until the age of 3 years. Plasma phenylalanine concentration was more than $3 \mathrm{mmol} / 1$. He responded well to dietary therapy initially, but the control was poor because of 
social problems. $\mathrm{He}$ is now severely retarded at the age of 9 years. Patient LGL5037, born in 1991, was diagnosed with plasma phenylalanine levels above $3 \mathrm{mmol} / 1$ at 7 months and responded well to dietary treatment. At the age of 4 years, the patient is considered to have mild developmental delay.

\section{HAPLOTYPE ANALYSIS}

Genomic DNA was isolated from blood leucocytes using standard procedures. RFLP analysis was performed by digestion of the DNA with a panel of seven restriction endonucleases, followed by Southern blot analysis using a full length PAH cDNA as hybridisation probe. ${ }^{8}$ Haplotypes of individual chromosomes were established as previously described. ${ }^{9}$

\section{MUTATION ANALYSIS}

Mutations in the PAH gene were detected and identified according to previously described procedures. $^{1011}$ In brief, 13 genomic DNA fragments covering the entire coding region and all splice sites of the PAH gene were amplified by PCR and scanned for mutations by denaturing gradient gel electrophoresis (DGGE).$^{12}$ Fragments showing an altered electrophoretic band pattern, suggesting the presence of a mutation, were sequenced directly. Primers for PCR and sequencing were as previously described. ${ }^{11} 13$

\section{Results and discussion}

All eight independent mutant chromosomes from the four Finnish PKU patients and their parents were subjected to RFLP haplotype and mutation analyses at the PAH locus. Comprehensive PCR/DGGE based mutation scanning of the coding sequence and all splice sites of the PAH gene led to the detection of a disease causing mutation on seven of the mutant alleles. Four different mutations were identified by direct sequence analysis (table). All patients carried the R408W mutation on one of their chromosomes. In all cases, the R408W mutation was associated with RFLP haplotype 2. The three remaining mutations were each different: the IVS2nt1 mutation was found on a haplotype 11 background, the IVS7nt 1 mutation on haplotype 4, and the R261Q mutation on haplotype 1 . No disease causing mutation was found on the haplotype 1 chromosome of patient LGL5072. We have previously achieved $100 \%$ mutation detection efficiency and $98 \%$ diagnostic efficiency by using the DGGE technique, ${ }^{1011}$ suggesting that the remaining disease

PAH mutations and associated RFLP haplotypes in Finnish PKU patients

\begin{tabular}{lll}
\hline Patient ID & Allele 1 & Allele 2 \\
\hline LGL5044 & R408W (HT 2) & IVS7nt (HT 4) \\
LGL5072 & R408W (HT 2) & ND (HT 1) \\
LGL5741 & R408W (HT 2) & R261Q (HT 1) \\
LGL5037 & R408W (HT 2) & IVS2nt (HT 11) \\
\hline
\end{tabular}

$\mathrm{HT}=$ haplotype

$\mathrm{ND}=$ not determined causing mutation in patient LGL5072 may be located outside the coding regions and splice sites of the PAH gene.

The most common mutation in Finland is R408W, accounting for half of the mutant chromosomes. This mutation, first described by DiLella $e t a l,{ }^{14}$ is one of the most frequent causes of PKU in Europe. Recent studies have shown that the R408W mutation clusters in two regions, north western Europe with the highest frequency reported in Ireland, and eastern Europe with the highest frequency reported in Lithuania. ${ }^{2}$ A detailed study of the association between R408W and RFLP haplotypes has shown that R408W is strongly associated with haplotype 1 in north western Europe, whereas it is strongly associated with haplotype 2 in eastern Europe. This led to the suggestion that R408W has two independent origins in Europe, one "Celtic" and one "Slavic". ${ }^{2}$ Extensive examination of the association between R408W and polymorphic VNTR and STR sites at the PAH locus supports this hypothesis. ${ }^{12}$

The R261Q mutation has been found in most European populations at relatively low frequencies, except for Switzerland and Turkey where it accounts for $32 \%$ and $10 \%$ of the mutant PAH chromosomes, respectively. ${ }^{15}$ RFLP and VNTR haplotype studies have shown that the R261Q mutation is nearly exclusively linked to a single haplotype 1 allele, suggesting that the R261Q mutation arose through a single founding event, probably in Switzerland, and subsequently spread to other populations. The IVS7nt 1 mutation has been found at very low relative frequencies (accounting for less than $2 \%$ of mutant PAH alleles) in Italy, Sicily, Spain, and Denmark, ${ }^{16}$ and no data indicating its origin have been reported. The IVS2nt 1 mutation has been found only in Palestinian Arabs, where it is linked to RFLP haplotypes 7 and $42 .{ }^{17}$ Its detection on one chromosome in Finland might seem surprising; however, it is carried by the patient's father who is a recent Palestinian immigrant. We note with interest that this mutation exceptionally occurs on haplotype 11 .

How do these findings relate to the extremely low frequency of PKU in Finland and to the origins of the Finnish population? The predominance of R408W (on four of eight mutant alleles) is fully consistent with the fact that this mutation is common in all of Finland's neighbours, for example, Sweden to the west $(22 \%)$, Lithuania to the south (69\%), and St Petersburg to the east $(67 \%){ }^{2}$ The fact that all four R408W mutations occur on haplotype 2 is again consistent with the corresponding proportions in Sweden (92\%), Lithuania $(100 \%)$, and St Petersburg (100\%). These findings, together with the occurrence of other single mutations whose origins are scattered, is consistent with the present day Finnish gene pool being of a general European nature with a strong "Baltic" component. ${ }^{18-21}$

The extremely low incidence of PKU mutations in Finland is best explained by a combination of founder effect, population bottlenecks, and genetic drift, that is, by chance. That a negative founder effect could 
alone account for the low incidence is illustrated by the fact that Finland's present population of 5 million is the result of the expansion of an extremely small founding population some 2000 years ago and that very little immigration has occurred thereafter. If, as discussed below, the "founders" numbered only, for example, 1000, then different numbers of mutation carriers (for example, one versus ten) among the founders would lead to very different present day frequencies even without considering bottlenecks and genetic drift. Other mendelian disorders with remarkably low incidences in Finland include cystic fibrosis, TaySachs disease, galactosaemia, Gaucher disease, and several others. ${ }^{21}$

Conversely, unusually high mutation frequencies for as many as 30 rare mendelian disorders occur in the Finnish population. ${ }^{22}$ Recent molecular studies have shown that, as expected under the founder hypothesis, a single ancestral founding mutation is usually responsible for the great majority of present day mutation carrying chromosomes. ${ }^{21}$ Moreover, linkage disequilibrium studies in relation to genetic distance around the gene for diastrophic dysplasia have suggested that the rough estimate of some 1000 founders some 2000 years ago having contributed the bulk of today's chromosomes may not be very far off. ${ }^{2324}$

The distribution of PKU mutations described here is consistent with there having been a very low frequency of early R408W founder chromosomes. In addition, there is a scattering of other mutations that have been contributed by the few immigrants that have arrived in Finland after the "founding".

We thank Drs Erik Malm, Pekka Mölsä, Taina Rautaoja, and We thank Drs Erik Malm, Pekka Mölsä, Taina Rautaoja, and
Jarmo Visakorpi for valuable information. PG is supported by Fellowship 99-9903 from The Danish Research Academy. This
study was supported by The Danish Medical Research Council (12-0058, 12-9292, 12-9744, and 12-1450), EC Programme BIOMED 1 (Area 3: Human Genome Analysis), The Danish Biotechnological Research and Development Programme 19911995 (Grant 5.18.03), The Danish Health Insurance Foundation ( $\mathrm{H} \mathrm{11} / 210-89, \mathrm{H} 11 / 282-90$, and $\mathrm{H} 11 / 257-91)$, The Novo Foundation, The Foundation of 1870, Franz Hoffmann's Memorial Fund, Emil C Hertz's Fund, The Academy of Finland, and The Folkhälsan Institute of Genetics.

1 Bycke S, Morgan K, Tyfield L, Dworniczak B, Scriver $\mathrm{CR}$. Evidence for origin, by recurrent mutation, of the phenylalanine hydroxylase R408W on two haplotypes in
European and Quebec populations. Hum Mol Genet 1994; 3:1675-7.

2 Eisensmith RC, Goltsov AA, O'Neill C, et al. Recurrence of the R408W mutation in the PAH locus in Europeans. Am $\mathcal{F}$ Hum Genet 1995;56:278-86.

3 Malm E. Oligophrenia phenylpyrouvica. Finska Läk Sällsk Handl 1950;92:221-31.

4 Palo J. Prevalence of phenylketonuria and some other metabolic disorders among mentally retarded patients in Finbolic disorders among mentally retarded
land. Acta Neurol Scand 1967;43:573.

5 Visakorpi JK, Palo J, Renkonen OV. The incidence of PKU in Finland. Acta Paediatr Scand 1971;60:666-8

6 Jervis GA. Phenylpyruvic oligophrenia (phenylketonuria) Ass Res Nerv Dis Proc 1954;33:259.

7 Bickel H, Bachmann C, Reckers R, et al. Neonatal mass screening for metabolic disorders. Eur $\mathcal{F}$ Pediatr 1981;137 133-9.

8 Lidsky AS, Ledley FD, DiLella AG, et al. Extensive restriction site polymorphism at the human phenylalanine
hydroxylase locus and application in prenatal diagnosis of phenylketonuria. Am f Hum Genet 1985;37:619-34.

9 Chakraborty R, Lidsky AS, Daiger SP, et al. Polymorphic DNA haplotypes at the human phenylalanine hydroxylase locus and their relationship with phenylketonuria. Hum Genet 1987;76:40-6.

10 Guldberg P, Henriksen KF, Güttler F. Molecular analysis of phenylketonuria in Denmark: $99 \%$ of the mutations of phenylketonuria in Denmark: $99 \%$ of the mutations omics 1993;17:141-6.

11 Guldberg P, Romano V, Ceratto N, et al. Mutational spectrum of phenylalanine hydroxylase deficiency in Sicily: implications for diagnosis of hyperphenylalaninemia in Southern Europe. Hum Mol Genet 1993;2:1703-7.

12 Abrams ES, Stanton VP. Use of denaturing gradient ge electrophoresis to study conformational transitions in nucleic acids. Methods Enzymol 1992;212:71-104.

13 Guldberg P, Mallmann R, Henriksen KF, Güttler F. Phenylalanine hydroxylase deficiency in a population in Germany: mutational profile and nine novel mutations. Hum Mutat (in press).

14 DiLella AG, Marvit J, Brayton K, Woo SLC. An aminoacid substitution involved in phenylketonuria is in linkage disequilibrium with DNA haplotype 2. Nature 1987:327: 333-6.

15 Eisensmith RC, Okano Y, Dasovich M, et al. Multiple origins of phenylketonuria in Europe. Am $\mathcal{f}$ Hum Genet origins of phenylk

16 Scriver CR, Hoang L, Byck S, Prevost L, eds. PAH Mutation Analysis Consortium. October 1994 Edition. Montreal: Analysis Consortium. Oct

17 Kleiman S, Bernstein J, Schwartz G, et al. A defective splice site at the phenylalanine hydroxylase gene in phenylketonuria and benign hyperphenylalaninemia among Palestinian Arabs. Hum Mutat 1992;1:340-3.

8 Nevanlinna HR. The Finnish population structure. Hereditas 1972;71:195-236.

19 Nevanlinna HR. Genetic aspects of rare neurological disease. Acta Neurol Scand 1978;57(suppl 67):37-55.

20 Workman PL, Mielke JH, Nevanlinna HR. The genetic structure of Finland. Am $\mathcal{}$ Phys Anthropol 1976;44:341-67.

21 de la Chapelle A. Disease gene mapping in isolated human populations: the example of Finland. F Med Genet 1993; 30:857-65.

22 Norio R, Nevanlinna HR, Perheentupa J. Hereditary diseases in Finland; rare flora in rare soil. Ann Clin Res 1973; 5:109-41.

23 Hästbacka J, de la Chapelle A, Kaitila I, et al. Linkage disequilibrium mapping in isolated founder populations disequilibrium mapping in isolated founder populations: 204-11.

24 Hästbacka J, de la Chapelle A, Mahtani MM, et al. The diastrophic dysplasia gene encodes a novel sulfate transporter: positional cloning by fine-structure linkage disequilibrium mapping. Cell 1994;78:1073-87. 